\title{
Identification of the Charge Carriers in Cerium Phosphate Ceramics
}

\author{
Hannah L. Ray ${ }^{a, b}$, L. C. De Jonghe ${ }^{a, b}$ \\ ${ }^{a}$ Materials Science Division, Lawrence Berkeley National Laboratory, Berkeley, \\ California, 94720, USA \\ ${ }^{\mathrm{b}}$ Department of Materials Science and Engineering, University of California, Berkeley, \\ Berkeley, California, 94720, USA
}

The total conductivity of Sr-doped cerium orthophosphate changes by nearly two orders of magnitude depending on the oxygen and hydrogen content of the atmosphere. The defect model for the system suggests that this is because the identity of the dominant charge carrier can change from electron holes to protons when the sample is in equilibrium with air vs. humidified hydrogen. In this work are presented some preliminary measurements that can help to clarify this exchange between carriers. The conduction behavior of a $2 \%$ Sr-doped $\mathrm{CePO}_{4}$ sample under symmetric atmospheric conditions is investigated using several techniques, including $\mathrm{AC}$ impedance, H/D isotope effects, and chronoamperometry.

\section{Introduction}

In a material that can conduct two different types of carriers, it is important to quantify the relative concentrations of the carriers when it is in equilibrium with a series of different atmospheres. If the variation of the carrier concentration with atmospheric content is known, the interaction between the carriers, or the effect one can have on the other's mobility, can be elucidated from conductivity measurements. In this study are presented the results of some preliminary experiments for identifying and determining the interactions between charge carriers in the $2 \% \mathrm{Sr}$-doped cerium orthophosphate $\left(\mathrm{CePO}_{4}\right)$ material system.

In past studies of the proton-conducting properties of $\mathrm{Sr}$-doped $\mathrm{CePO}_{4}$, it has been observed that the material's conductivity changes by two orders of magnitude in oxidizing vs. reducing conditions $[1,2]$. This large change in conductivity is caused by the dominance of different charge carriers in different atmospheres. Defect models propose that electron holes are the dominant carrier in oxidizing conditions, whereas in reducing conditions, protons dominate. In phosphate materials, oxygen vacancies are manifested as pyrophosphate links [3].

$$
\mathrm{V}^{\circ} \mathrm{O}+2 \mathrm{PO}_{4}{ }^{\mathrm{X}} \mathrm{PO} 4 \rightarrow \mathrm{P}_{2} \mathrm{O}_{7}^{\circ}{ }_{(2 \mathrm{PO} 4)}
$$

Where $\mathrm{V}^{\circ} \mathrm{O}$ is an oxygen vacancy, $\mathrm{PO}_{4}{ }^{\mathrm{X}}{ }_{\mathrm{PO} 4}$ is a phosphate tetrahedron on its proper site, and $\mathrm{P}_{2} \mathrm{O}_{7}$ (2PO4) represents two phosphate tetrahedra sharing an oxygen corner.

Oxygen vacancies are generally created either by acceptor doping or native Schottky disorder induced during the high temperature synthesis. These oxygen vacancies can be compensated by incorporation of either a proton or an electron hole. 


$$
\begin{aligned}
& \mathrm{P}_{2} \mathrm{O}_{7}{ }_{(2 \mathrm{PO} 4)}^{\circ}+1 / 2 \mathrm{O}_{2} \rightarrow 2 \mathrm{PO}_{4}{ }_{(2 \mathrm{PO} 4)}^{\mathrm{X}}+2 h^{\circ} \\
& \mathrm{P}_{2} \mathrm{O}_{7}{ }^{\circ}{ }_{(2 \mathrm{PO} 4)}+\mathrm{H}_{2} \mathrm{O} \rightarrow 2 \mathrm{HPO}_{4}{ }_{\mathrm{PO} 4}
\end{aligned}
$$

Where $h^{\circ}$ represents an electron hole, and $\mathrm{HPO}_{4}{ }_{\mathrm{PO} 4}$ represents a proton bound to an oxygen on a phosphate tetrahedron.

In the $\mathrm{CePO}_{4}$ system, electron holes have an additional means of incorporation via the ability of the cerium cation to exist in two valence states: $\mathrm{Ce}^{3+}$ and $\mathrm{Ce}^{4+}$.

$$
\left(\mathrm{Ce}^{3+}\right)^{\mathrm{X}}{ }_{\mathrm{Ce} 3+}+\mathrm{h}^{\circ} \rightarrow\left(\mathrm{Ce}^{4+}\right)_{\mathrm{Ce} 3+}^{\circ}
$$

Where $\left(\mathrm{Ce}^{3+}\right)^{\mathrm{X}}{ }_{\mathrm{Ce} 3+}$ represents a cerium $3+$ cation residing on its proper site, and $\left(\mathrm{Ce}^{4+}\right)_{\mathrm{Ce} 3+}$ represents a cerium $4+$ cation residing on a cerium $3+$ site.

Recent computational experiments on a structurally similar material, Ba-doped lanthanum orthophosphate, have shown that the energy surface for a protonic carrier has an energy well, or "trap" near the dopant atoms due to Coulombic forces between the positively charged carrier and the effective negative charge near the acceptor dopant [4]. If protons and holes coexist in a material, it could be possible for them to exchange with each other and free protons from their traps, increasing protonic mobility.

$$
(\mathrm{SrH})^{\mathrm{X}}{ }_{\mathrm{Ce} 3+}+h^{\circ} \rightarrow(\mathrm{Srh})^{\mathrm{X}}{ }_{\mathrm{Ce} 3+}+\mathrm{H}^{\circ}
$$

To verify the proposed defect model, and determine the carrier concentration's dependence on the atmospheric conditions, several different electrochemical characterization techniques can be used. Two general types of experimental setup can be identified: those which expose both sides of the membrane to the same atmosphere (symmetric conditions), and those which expose the two sides of the membrane to different atmospheres (asymmetric conditions.) Symmetric conditions allow the determination of material properties of the membrane under controlled atmosphere. Tests under asymmetric conditions better simulate the applications for the material (gas separation membranes) and also allow determination of transference numbers and hydrogen permeation rates. However, if the gradient in chemical potential is too large, the proton transference number can change as a function of position within the membrane; the data analysis of these types of tests must take this into account.

The experimental techniques presented in this work all use symmetric atmospheric conditions. The total conductivity of $2 \% \mathrm{Sr}$-doped $\mathrm{CePO}_{4}$ is measured using $\mathrm{AC}$ impedance techniques in ambient air, wet hydrogen, and wet deuterium atmospheres. Chronoamperometric techniques are used to determine the I-V curve for the sample in equilibrium with the air and with $4 \% \mathrm{H}_{2}$ in Ar.

\section{$\underline{\text { Synthesis }}$}

\section{Experimental Techniques}

To prepare the $\mathrm{CePO}_{4}$ powders, a $2 \mathrm{M}$ solution of $\mathrm{NH}_{4} \mathrm{H}_{2} \mathrm{PO}_{4}$ (Acros Organics, $99.9+\%$ ) was added dropwise to a $2 \mathrm{M}$ aqueous solution of $\mathrm{Ce}\left(\mathrm{NO}_{3}\right)_{3} \cdot 6 \mathrm{H}_{2} \mathrm{O}$ (Alfa Aesar, 99.999\% metals basis). The resulting $\mathrm{CePO}_{4}$ precipitate was washed with water, filtered, and then calcined at $800^{\circ} \mathrm{C}$ for 2 hours to transform it from the rhabdophane to the monoclinic phase. The resulting powders were off-white in color. 
To prepare the doped samples, $\mathrm{CePO}_{4}$ and 2 at. $\% \mathrm{SrHPO}_{4}$ (Sigma Aldrich) powders were ball-milled for 24 hours in isopropyl alcohol with $2 \mathrm{wt}$ \% polyvinyl butyral covinyl alcohol-co-vinyl acetate (Sigma-Aldrich), dibutyl phthalate (Mallinckrodt OR), and menhaden fish oil (Sigma-Aldrich). The powder mixture was dried and sieved through 325 mesh. $1 \frac{1}{2}$ inch diameter pellets were pressed at $10 \mathrm{kpsi}$. Pellets were sintered for 5 hours at $1200^{\circ} \mathrm{C}$. Sintered pellets were green in color.

\section{Structural Characterization}

X-ray diffraction (XRD) of powders and sintered pellets was carried out on a Philips PW3040 X'Pert Pro Diffractometer using $\mathrm{Cu} K \alpha(\lambda=1.5406 \AA)$ source operated with a $45 \mathrm{keV} \mathrm{X}$-ray tube voltage. The microstructure and chemistry of the pellets were studied using an analytical scanning electron microscope (SEM, Zeiss Gemini Ultra-55) operated between 5 and $30 \mathrm{kV}$.

\section{Electrochemical Characterization}

\section{Electrodes}

Platinum paste (Heraeus) was used to attach platinum mesh to either side of each pellet. Pellets were fired at $900^{\circ} \mathrm{C}$ for 1 hour to cure the paste.

\section{Test Rig for Symmetric Atmospheric Conditions}

To measure the conduction behavior of the sample in controlled atmospheres at elevated temperatures, it was necessary to build an enclosed test rig, with ports for gas flow and wire contacts, which placed the sample in the center of a tube furnace. A home designed aluminum cap with holes for gas in, gas out, and wire contacts was fabricated. The ends of a 12" and a 6" single bore 0.15 " diameter alumina tube (CoorsTek) were cemented into the gas line holes in the aluminum cap. Platinum wires were fed through 12 " long double bore 0.15 " diameter alumina tubes, and the ends of these tubes were also cemented into the aluminum cap. The sample's electrical contacts were wound around the ends of the wires, suspending the sample at the ends of the 12" tubes. The 12" tube for gas in pointed directly at the sample. A stainless steel Swagelok 1" union Tube fitting was used to grip the alumina cap, and the other side of the union tube fitting gripped a closed end 1" diameter alumina tube that fit over the gas tubes, electrical contacts, and sample. The sample end of the rig was placed in the center of a tube furnace, while the aluminum cap sat at room temperature. The potentiostat contacts and gas lines were attached to the appropriate ports of the alumina cap.

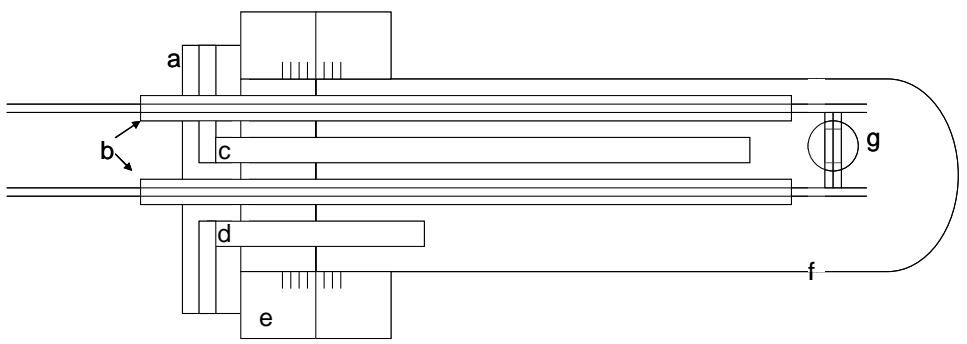

Figure 1. Test rig for electrochemical measurements in controlled atmosphere (symmetric). (a) Aluminum cap. (b) Double bore alumina tubes with Pt wire fed through to contact the sample to the potentiostat. (c) Tube for gas in and (d) tube for gas out are cemented into aluminum cap. (e) Swagelok ?? grips alumina cap and screws into other half which grips (f) Coorstek closed end tube. (g) Sample attaches to the Pt wires. 


\section{AC Impedance Measurements}

The total conductivity of the samples was measured using a Bio-Logic potentiostat/galvanostat/EIS with SP-150 VSP/VMP3 boosters. The frequency was varied from $1 \mathrm{MHz}$ to $0.1 \mathrm{~Hz}$, and the alternating voltage amplitude was $50 \mathrm{mV}$. Conductivities were measured between 350 and $600^{\circ} \mathrm{C}$ in ambient air, $\mathrm{H}_{2}$ gas bubbled through water, and $\mathrm{D}_{2}$ gas bubbled through $\mathrm{D}_{2} \mathrm{O}$. Impedance data was processed using EC-Lab (BioLogic).

\section{Chronoamperometry Measurements}

The same Bio-Logic potentiostat/galvanostat and EC-Lab software were used for the chronoamperometry measurements. To determine the I-V behavior of the sample, the potential was stepped from $0.05 \mathrm{~V}$ to $1.4 \mathrm{~V}$ in increments of $0.05 \mathrm{~V}$ and held for 2 minutes per step, with a rest period of 2 minutes at open circuit voltage. The current was monitored during this time. Since the time increments were kept constant, the linearity of the I-V curve could be evaluated directly from the current vs. time plot. This experiment was carried out at $500^{\circ} \mathrm{C}$ in ambient air, as well as in a gas flow of $4 \% \mathrm{H}_{2}$ in Argon bubbled through $\mathrm{H}_{2} \mathrm{O}$.

\section{Structure}

\section{Results and Discussion}

Figure $2 \mathrm{a}$ shows the XRD spectrum of the $2 \% \mathrm{Sr}$-doped $\mathrm{CePO}_{4}$ sintered pellet. The crystal structure corresponds to monoclinic $\mathrm{CePO}_{4}$ (JCPDS card 32-0199) with no extra peaks. Figure $2 \mathrm{~b}$ is an SEM image of a fracture surface of the sample which shows grains ranging from 5 to 20 microns in length, with some trapped pores. Grains are not densely packed; some porosity exists between grains as well. These images of the microstructure explain the low density ( $86 \%$ of theoretical) measured for this sample. When the density of a ceramic is this low, it is possible for there to be chains of pores which extend through the thickness of the sample. Therefore, this particular sample is not appropriate for a gas separation membrane. It can only be used for measurements in symmetric atmospheres.

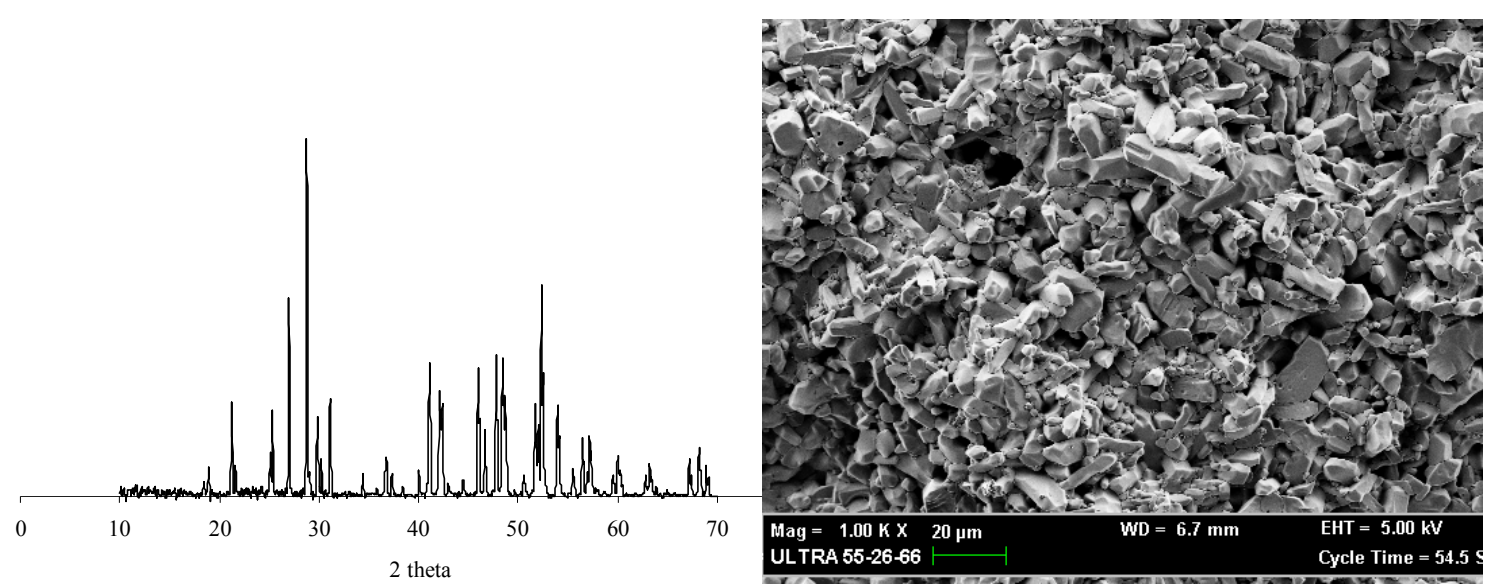

Figure 2. Structure of $2 \%$ Sr-doped $\mathrm{CePO}_{4}$. (a) The XRD pattern of the sintered $\mathrm{CePO}_{4}$ pellet matches JCPDS card 32-0199 for monazite. (b) The fracture surface image shows that the grain size varies from 5 to 20 microns, and there are intragranular as well as intergranular pores evident.

$\underline{\text { Total conductivity in symmetric, oxidizing conditions }}$ 
The total conductivity of the $2 \%$ Sr-doped $\mathrm{CePO}_{4}$ sample was measured in air using AC impedance. As shown in Figure 3, the conductivity varies from $10^{-4.9} \mathrm{~S} / \mathrm{cm}$ at $350^{\circ} \mathrm{C}$ to $10^{-3.1} \mathrm{~S} / \mathrm{cm}$ at $600^{\circ} \mathrm{C}$, with activation energy $0.82 \mathrm{eV}$. This is comparable to the values found in the literature. [1,2]. When air was bubbled through $\mathrm{H}_{2} \mathrm{O}$ vs. $\mathrm{D}_{2} \mathrm{O}$, no difference in conductivity was observed. This confirms that the dominant carrier when the sample is in equilibrium with moist air is not protons. It is predicted from the defect model for this material that the carrier under these conditions is electron holes. A thermopower measurement could reveal the sign of the carrier; results from this type of experiment will be published in a future work.

The I-V curve for the sample at $500^{\circ} \mathrm{C}$ in equilibrium with the ambient air as determined by the chronoamperometric experiment is shown in Figure 4a. The I-V curve is linear, which indicates that the sample under these conditions behaves as a perfect resistor with a resistivity of $1500 \Omega / \mathrm{cm}$.

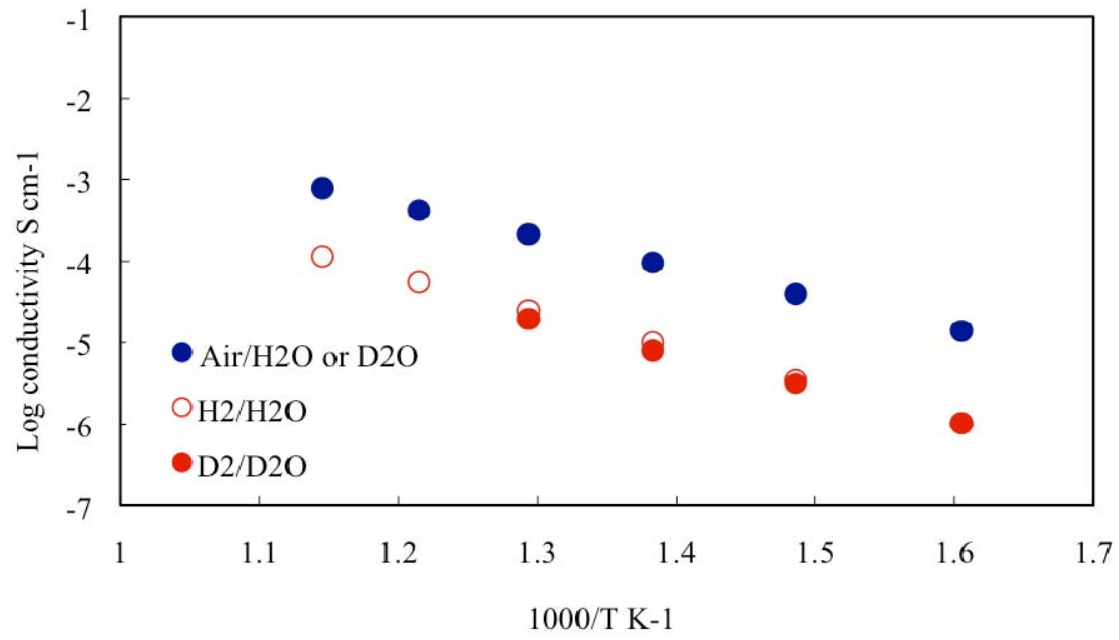

Figure 3. Total conductivity of $2 \%$ Sr-doped $\mathrm{CePO}_{4}$ vs. $1000 / \mathrm{T}$ at temperatures between $350^{\circ} \mathrm{C}(1000 / \mathrm{T}=1.6)$ and $600^{\circ} \mathrm{C}(1000 / \mathrm{T}=1.15)$ as measured by $\mathrm{AC}$ impedance in air, $\mathrm{H}_{2}$ gas bubbled through $\mathrm{H}_{2} \mathrm{O}$, and $\mathrm{D}_{2}$ gas bubbled through $\mathrm{D}_{2} \mathrm{O}$. The conductivity in air is approximately 2 orders of magnitude higher than the conductivity in reducing atmospheres.

Total conductivity in symmetric, reducing conditions

The total conductivity of the $2 \%$ Sr-doped $\mathrm{CePO}_{4}$ sample was measured in $\mathrm{H}_{2}$ gas bubbled through room temperature $\mathrm{H}_{2} \mathrm{O}$, which gives a relative humidity of about $3 \%$. Figure 3 shows that the conductivity varied from $10^{-6} \mathrm{~S} / \mathrm{cm}$ at $350^{\circ} \mathrm{C}$ to $10^{-4} \mathrm{~S} / \mathrm{cm}$ at $600^{\circ} \mathrm{C}$ with an activation energy of $0.92 \mathrm{eV}$. The conductivity of the sample in $\mathrm{D}_{2}$ gas bubbled through $\mathrm{D}_{2} \mathrm{O}$ was lower by a factor of 1.4. This indicates that the dominant carrier in reducing conditions is protons.

The I-V curve for the sample at $500^{\circ} \mathrm{C}$ in equilibrium with an $3 \%$ humid gas consisting of $4 \% \mathrm{H}_{2}$ in Argon is linear, as shown in Figure $4 \mathrm{~b}$. This indicates that the sample under these conditions behaves as a perfect resistor, with resistivity 277,000 $\Omega / \mathrm{cm}$.

The chronoamperometric behavior of the sample under transient conditions (for example, during equilibration after a step change in the composition of the atmosphere) is expected to give nonlinear I-V behavior, with a current that varies with potential depending on the applied potential and temperature. Results from transient experiments of this type will be reported in a future work. 

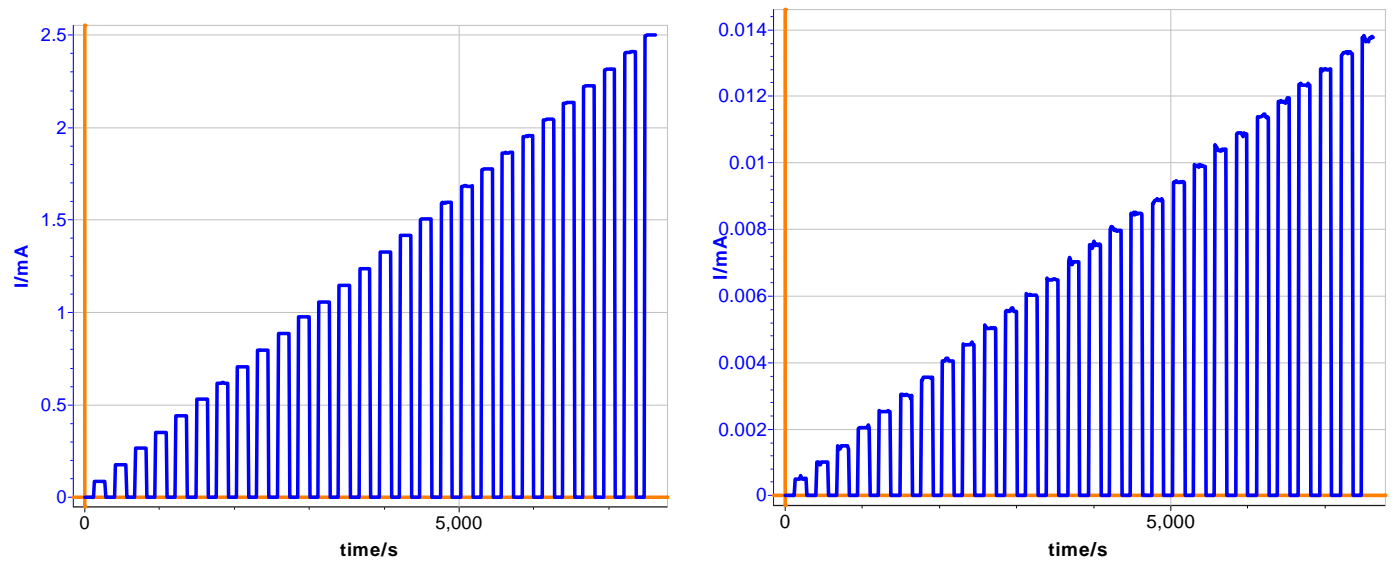

Figure 4. Plots of current vs. time when the $2 \%$ Sr-doped $\mathrm{CePO}_{4}$ sample at $500^{\circ} \mathrm{C}$ is subjected to potentials between $0.05 \mathrm{~V}$ and $1.4 \mathrm{~V}$, increasing in increments of $0.05 \mathrm{~V}$ held for 2 minutes with a 2 minute rest period between each step. (a) In air, the maximum current is nearly $2.5 \mathrm{~mA}$. (b) In $4 \% \mathrm{H}_{2}$ in Argon at 3\% humidity, the maximum current is $0.014 \mathrm{~mA}$. Both I-V curves are linear, as both samples are in equilibrium with the atmosphere.

\section{Conclusion}

Ceramic samples of monoclinic $2 \%$ Sr-doped $\mathrm{CePO}_{4}$ are prepared by precipitation of cerium nitrate and ammonium hydrogen phosphate, followed by standard ceramic processing techniques. The conductivity between 350 and $600^{\circ} \mathrm{C}$ as measured by $\mathrm{AC}$ impedance is shown to vary from $10^{-5}$ to $10^{-3} \mathrm{~S} / \mathrm{cm}$ in air, and from $10^{-6}$ to $10^{-4} \mathrm{~S} / \mathrm{cm}$ in humidified $\mathrm{H}_{2}$ gas. $\mathrm{H} / \mathrm{D}$ isotope effects show that the dominant carrier in reducing conditions is protons, while in air it is not protons. The I-V curves of the samples as determined by chronoamperometric techniques are linear when they are in equilibrium with air or $4 \% \mathrm{H}_{2}$ in Argon.

Future works will show the results of more of these measurements in symmetric conditions, under atmospheres in which the $\mathrm{pO}_{2}$ and $\mathrm{pH}_{2}$ are systematically varied. The chronoamperomentric measurement technique will be used to evaluate the equilibrium of the sample with the atmosphere. Chronoamperometry will also be used to monitor the change in conductivity behavior real time under nonequilibrium conditions. This type of measurement technique, paired with emf measurements with small concentration gradients applied, can help to elucidate the interaction between protonic and electronic carriers in the ceramic.

\section{Acknowledgments}

This research was supported by the Director, Office of Science, Office of Basic Energy Sciences, Materials Sciences and Engineering Division, of the U.S. Department of Energy under Contract No. DE-AC02-05CH11231. H. L. Ray would like to acknowledge the National Defense Science and Engineering Graduate Fellowship. The authors acknowledge support and use of the Ultra-55 SEM facility at the Molecular Foundry at Lawrence Berkeley National Laboratory. 


\section{References}

1. N. Kitamura, K. Amezawa, Y. Tomii, T. Hanada, N. Yamamoto, T. Omata, S. Otsuka-Yao-Matsuo, Journal of the Electrochemical Society, 152 (4) A658-A663 (2005).

2. E. Gomez del Moral, D. P. Fagg, E. Chinarro, J. C. C. Abrantes, J. R. Jurado, G. C. Mather, Ceramics International, 35, 1481-1486 (2005).

3. K. Amezawa, H. Maekawa, Y. Tomii, N. Yamamoto, Solid State Ionics, 145, 233240 (2001).

4. N. Adelstein, J. Chem. Phys., submitted. 